\title{
Dysphagia Causes Symptom Fluctuations after Oral L-DOPA Treatment in a Patient with Parkinson Disease
}

\author{
Hiromasa Sato $^{a}$ Toshiyuki Yamamoto $^{a}$ Masako Sato $^{b}$ \\ Yoshihiko Furusawa ${ }^{a}$ Miho Murata $^{a}$ \\ aDepartment of Neurology, National Center Hospital, National Center of Neurology and \\ Psychiatry, Tokyo, Japan; bepartment of Rehabilitation Medicine, National Center \\ Hospital, National Center of Neurology and Psychiatry, Tokyo, Japan
}

\section{Keywords}

Parkinson disease - No-on phenomenon - Gastrointestinal tract · Pharmacologic actions ·

Rehabilitation

\begin{abstract}
Objective: The causes of "delayed-on" and "no-on" phenomena in Parkinson disease (PD) are thought to have some impact on the progress of L-DOPA from the time of ingestion until it reaches the brain and is converted to dopamine. Dysphagia can cause fluctuating symptom expression in L-DOPA therapy for PD. Case Description: A 69-year-old man with PD presented with "delayed-on" and "no-on" phenomena. The patient developed a gait disorder at age 60 years, and he began coughing on his food during breakfast at age 64 years. Even though he was independent in daily life, he could not eat because of dysphagia in an "off" state. Videofluoroscopic examination of swallowing in an "off" state revealed bradykinesia of the tongue and the retention of tablets in the epiglottic vallecula. We trained him to keep his tongue in strong contact with the upper incisors before swallowing. After rehabilitation of dysphagia, the frequency of "delayed-on" and "no-on" phenomena decreased, and his peak L-DOPA plasma concentration was elevated. Additionally, transdermal rotigotine (RTG) was initiated at a maintenance dose of $9.0 \mathrm{mg}$. The patient reported improvement in swallowing,
\end{abstract}


and the frequency of "no-on" phenomena decreased. Conclusion: In PD patients, the "noon" phenomenon can be caused by posterior contractile dysfunction of the tongue, and it can be improved with training of the tongue and transdermal RTG administration.

\section{Introduction}

Dysphagia is thought to be caused by lingual bradykinesia. It becomes more frequent as Parkinson disease (PD) progresses, but studies with objective measures do not always show an association between dysphagia and disease duration or severity [1]. Dysphagia-related impairment of tablet transport through the digestive tract could easily impair L-DOPA absorption, but few reports have considered dysphagia as the cause of fluctuations in symptom expression known as "delayed-on" (a significant delay in response to a dose) and "no-on" (complete dose failure) phenomena. This case report describes a patient with PD in whom impaired transfer of medication in the oropharynx caused such symptom fluctuations.

\section{Case Description}

A 69-year-old man complained of difficulty swallowing his morning medication and of variation in the time required to reach an "on" state. He was previously diagnosed with internuclear ophthalmoplegia at the age of 64 years, which had resolved in 1 day. The family and lifestyle histories were unremarkable.

At 60 years of age, the patient's left leg began dragging when he walked, and at 62 years dysarthria developed. Oral administration of L-DOPA $100 \mathrm{mg} /$ carbidopa $10 \mathrm{mg}$ (LDCD) was initiated based on a diagnosis of PD, and walking and dysarthria improved. At 64 years, the patient regularly began to choke during breakfast, and by 65 years, wearing-off phenomena had begun. At 66 years, it became difficult for the patient to receive oral medication, and he began consuming his medication with jelly. Between 65 and 66 years, his weight decreased from $73 \mathrm{~kg}$ to $65 \mathrm{~kg}$. By 68 years, completing breakfast required 3-4 h. In the "on" state, the patient was independent in activities of daily living. At 69 years, LDCD $600 \mathrm{mg}$ and entacapone $600 \mathrm{mg}$ were each received in six portions, selegiline hydrochloride $10 \mathrm{mg}$ in two portions, and zonisamide $25 \mathrm{mg}$ and trihexyphenidyl hydrochloride $2 \mathrm{mg}$ in one portion. To assist swallowing, tablets were wrapped in a water-soluble film, but it remained easier for the patient to swallow the wrapped tablets with jelly. However, he sometimes later expelled the tablets through coughing. Doses were frequently accompanied by "delayed-on" or "noon" phenomena.

The patient's height was $162 \mathrm{~cm}$ and his weight was $55 \mathrm{~kg}$. There were no auscultatory abnormalities of the chest or abdomen. Mild right flexion was observed in the standing position. The Mini-Mental State Examination score was 29/30. No abnormalities in cranial nerves, muscle strength, or deep tendon reflexes were found. No pathological reflexes were present. Muscle rigidity was observed in the neck and all four limbs, and the patient was without tremor. He could walk independently in the "on" state, but experienced retropulsion in the "off" state. The Hoehn and Yahr classification was stage III in the "on" state and stage IV in the "off" state. The "off" state was characterized by strong akinesia and bradykinesia, sialorrhea, reduced vocal intensity, and poor speech intelligibility; all symptoms improved in the "on" state. 
There were no abnormalities in the general blood tests or on head magnetic resonance imaging (Fig. 1). A videofluorographic examination of swallowing (VFG) was performed in which the patient consumed $10 \mathrm{~mL}$ of barium-containing thickened water (Ba) and four tablets wrapped in a water-soluble film. VFG showed that the tongue barely moved, and the time required for the Ba to pass the lower border of the mandible and transfer to the pharynx was $27 \mathrm{~s}$ (typically $2 \mathrm{~s}$ in normal individuals). A swallowing reflex was induced after the Ba reached the pyriform sinuses. The backward movement of the tongue root was very limited, and the tablets remained in the epiglottic vallecula (Fig. 2). Respiratory status was unchanged, and no aspiration was observed. After consumption of the medication, $2 \mathrm{~h}$ were needed for the patient to reach the "on" state, at which point, tongue movement improved and nothing remained in the pharynx.

The patient was further examined with a L-DOPA test [2]. One tablet of LDCD was grounded and administered to the patient with jelly in a fasting state after he awakened in the morning. Blood levels of L-DOPA were measured from before the medicine was administered until $240 \mathrm{~min}$ afterward (seven time points), and symptoms of parkinsonism were assessed. The patient choked when swallowing the medicine and required suctioning during the L-DOPA test. There was no improvement in parkinsonian symptoms even $4 \mathrm{~h}$ after administration, and the blood L-DOPA level only rose to $0.78 \mathrm{nmol} / \mathrm{mL}$ (Fig. 3).

The patient was shown a video of his swallowing process and was informed that in the "off" state his lingual movement was insufficient to transfer food from his mouth to his pharynx. Tongue resistance training was then initiated. First, the patient held the plastic probe of the tongue pressure-measuring instrument $(25 \times 1.8 \mathrm{~mm})$ in his mouth. Then, he was asked to press the probe hard with his tongue tip. The probe was pressed between the tongue tip and the upper incisors for $5 \mathrm{~s}$ with maximum power, and the tongue pressure was measured. The patient performed this training five times before each meal. In addition, he was instructed to press his tongue tip to the palatal side of the upper incisors without the probe before swallowing.

The L-DOPA test was repeated 8 days after the training began. The patient swallowed the tablets quickly with jelly and reached the "on" state after $30 \mathrm{~min}$, with improvements in akinesia, bradykinesia, hypokinetic dysarthria, and swallowing. The highest blood level of the drug was $9.99 \mathrm{nmol} / \mathrm{mL} 30 \mathrm{~min}$ after administration (Fig. 3). The highest tongue pressure measured in the "on" state improved to $28.8 \mathrm{kPa}$ from $23.7 \mathrm{kPa}$ over 10 days. The frequency of reaching the "on" state $30 \mathrm{~min}$ after receiving the medicine increased from 2 times/week before training to 5 times/week after training. Additionally, transdermal rotigotine (RTG) was initiated at a maintenance dose of $9.0 \mathrm{mg}$. The patient reported improvement in swallowing, and the frequency of "no-on" phenomena decreased. The patient gained $3 \mathrm{~kg}$ 3 months after RTG treatment without edema.

\section{Discussion}

L-DOPA acts centrally but is converted to dopamine before reaching the brain. The primary causes of "delayed-on" and "no-on" phenomena in PD are thought to be delayed gastric emptying time and decreased absorption after ingestion of large amounts of amino acids [3, 4]. In addition, the efficacy of oral L-DOPA therapy is further degraded peripherally by enzymatic breakdown by amino acid decarboxylase and catechol-0-methyltransferase, and by competition from large, neutral amino acids for transport across the blood-brain barrier [5]. Although the possibility of impaired tablet transport resulting from dysphagia has been not- 
ed, it has not been investigated. Previously, we reported the case of a patient with PD, an esophageal hiatal hernia, and kyphosis, who experienced esophageal retention of oral medication and fluctuation of his parkinsonian symptoms [6]. In the present patient, tablets were retained in the epiglottic vallecula, and therefore, it was assumed that absorption of the tablets would be rather slow, destabilizing the effects of L-DOPA and also possibly reducing the peak plasma concentration of the drug.

L-DOPA therapy for dysphagia in patients with PD is considered relatively ineffective [7]; however, reports of improvements in the oral stage of swallowing with L-DOPA therapy [8] and RTG [9] have been published. The present patient could not receive oral L-DOPA in his "off" state, and as such, his lingual bradykinesia could not be treated. However, we treated him with transdermal RTG and successfully relieved his dysphagia and he gained $3 \mathrm{~kg}$. This case illustrates the successful use of RTG through the transdermal route to treat dysphagia in PD.

In PD, cognitive cueing (e.g., Lee Silverman Voice Treatment ${ }^{\circledR}$ ) is also effective for treating problems with swallowing [10]. The targeted training of swallowing-related sensorimotor actions may improve function to some degree in PD [11]. In cases of post-stroke dysphagia, 8 weeks of training to apply tongue pressure to the anterior and posterior portions of the hard palate produced increases in maximum tongue pressure and swallowing pressure and reduced retention in the epiglottic vallecula [12]. Such training in the present patient increased the maximum tongue pressure and improved the daily symptomatic fluctuation as well as blood L-DOPA levels. We consider it unlikely that the patient's lingual muscle strength was increased in such a short period and attribute the benefits of training to improved sensorimotor function.

\section{Conclusion}

This case supports the hypothesis that dysphagia may be a cause of "no-on" and "delayed-on" phenomena in patients with PD. It also highlights retention of tablets in the epiglottic vallecula, which may be overlooked because it cannot be assessed visually. After treatment via rehabilitation for dysphagia, our patient's peak L-DOPA plasma concentration was elevated 10 -fold and his symptom fluctuations improved. In patients with dysphagia for whom oral medication produces weak or unstable effects, it may be necessary to examine for retention in the epiglottic vallecula using VFG or laryngoscopy after the medicine has been administered.

\section{Acknowledgments}

This work was supported by an Intramural Research Grant (27-4) for Neurological and Psychiatric Disorders of NCNP, Japan.

\section{Statement of Ethics}

The patient provided oral informed consent for publication of this report and the study design was approved by the institutional ethics review board. 


\section{Disclosure Statement}

All authors have no competing interest.

\section{References}

1 Pfeiffer RF: Gastrointestinal dysfunction in Parkinson's disease. Lancet Neurol 2003;2:107-116.

-2 Murata M, Mizusawa H, Yamanouchi H, Kanazara I: Chronic levodopa therapy enhances dopa absorption: contribution to wearing-off. J Neural Transm 1996;103:1177-1185.

-3 Djaldetti R, Baron J, Ziv I, Melamed E: Gastric emptying in Parkinson's disease: patients with and without response fluctuations. Neurology 1996;46:1051-1054.

-4 Nutt JG, Woodward WR, Hammerstad JP, Cater JH, Anderson JL: The "on-off" phenomenon in Parkinson's disease. Relation to levodopa absorption and transport. N Engl J Med 1984;310:483-488.

5 Poewe W, Antonini A, Zijlmans JC, Burkhard PR, Vingerhoets F: Levodopa in the treatment of Parkinson's disease: an old drug still going strong. Clin Interv Aging 2010;5:229-238.

-6 Chihara N, Yamamoto T, Lin Y, Tsukamoto T, Ogawa M, Murata M: Severe kyphosis and esophagus hiatal hernia affected in the levodopa absorption of a patient with Parkinson's disease (in Japanese). Rinsho Shinkeigaku 2009;49:493-496.

7 Palhagen S, Lorefalt B, Carlsson M, Ganowiak W, Toss G, Unosson M, Granérus AK: Does L-dopa treatment contribute to reduction in body weight in elderly patients with Parkinson's disease? Acta Neurol Scand 2005;111:12-20.

8 Fuh JL, Lee RC, Wang SJ, L CH, Wang PN, Chiang JH, Liu HC: Swallowing difficulty in Parkinson's disease. Clin Neurol Neurosurg 1997;99:106-112.

$\$ 9$ Hirano M, Isono C, Sakamoto H, Ueno S, Kusunoki S, Nakamura Y: Rotigotine transdermal patch improves swallowing in dysphagic patients with Parkinson's disease. Dysphagia 2015;30:452-456.

10 Ransmayr G: Physical, occupational, speech and swallowing therapies and physical exercise in Parkinson's disease. J Neural Transm 2011;118:773-781.

11 Russell JA, Ciucci MR, Connor NP, Schallert T: Targeted exercise therapy for voice and swallow in persons with Parkinson's disease. Brain Res 2010;1341:3-11.

-12 Robbins J, Kays SA, Gangnon RE, Hind JA, Hewitt AL, Gentry LR, Taylor AJ: The effect of lingual exercise in stroke patients with dysphagia. Arch Phys Med Rehabil 2007;88:150-158. 


\section{Case Reports in Neurology}

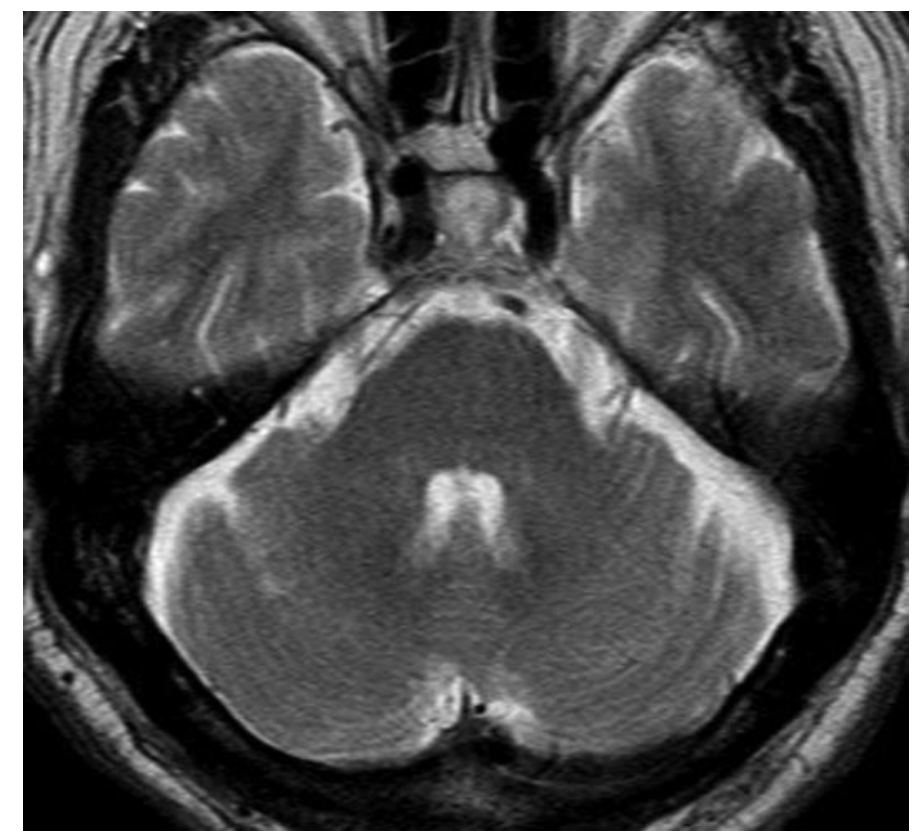

Fig. 1. Brain magnetic resonance imaging results. T2-weighted magnetic resonance imaging (axial image of the pons at the level of the medial longitudinal fasciculus) was normal in this patient.

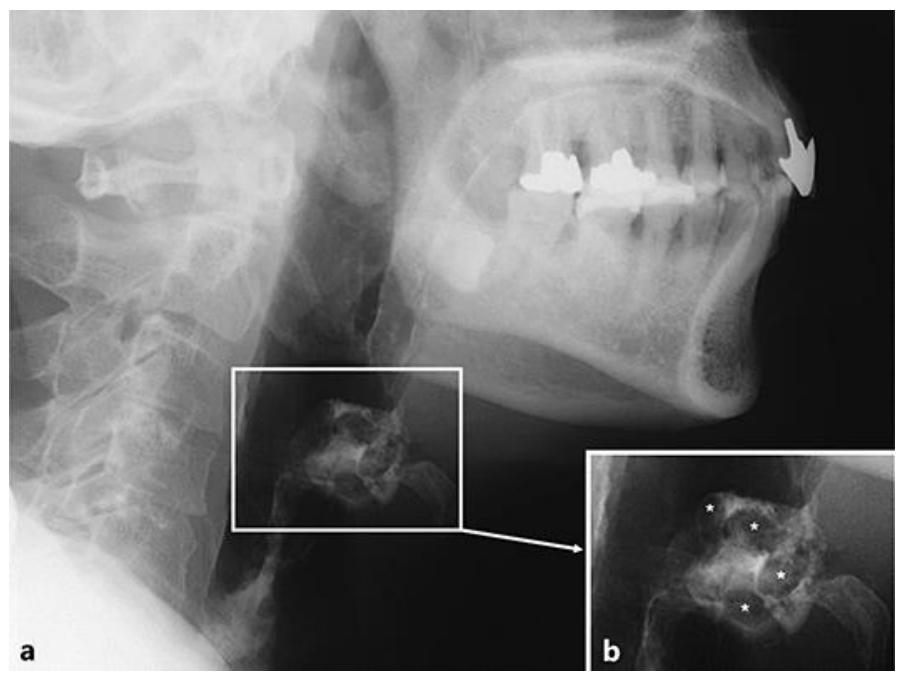

Fig. 2. Videofluoroscopic examination of the swallowing process. a Four tablets covered with a watersoluble film were administered orally in $10 \mathrm{~mL}$ of jelly containing barium. Tablets remained in the epiglottic vallecula (white frame). b Enlarged view of the epiglottic vallecula. The tablets remaining in the epiglottic vallecula are seen as filling defects (asterisks). 


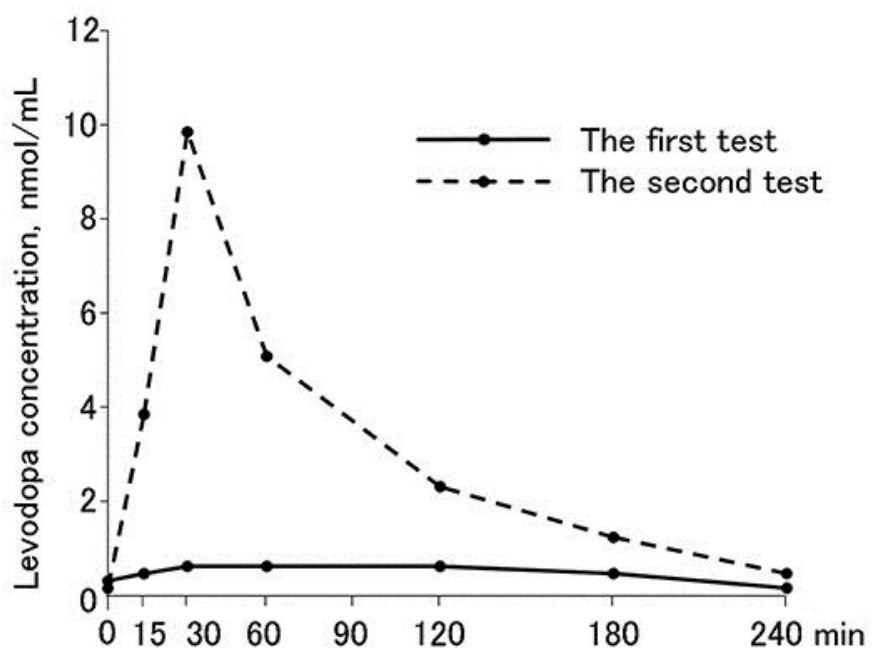

Fig. 3. Results of the L-DOPA test. One ground L-DOPA $100 \mathrm{mg} /$ carbidopa $10 \mathrm{mg}$ tablet was administered orally on an empty stomach after waking in the morning, and the L-DOPA blood concentration was measured seven times from before the tablet was administered to $240 \mathrm{~min}$ after ingestion. Prior to rehabilitative intervention, the tablet was ineffective (a "no-on" state), parkinsonian signs did not improve, and the maximum blood L-DOPA concentration was $0.78 \mathrm{nmol} / \mathrm{mL}$ (solid line). After dysphagia rehabilitation, the patient was able to swallow the tablets quickly, his parkinsonism symptoms improved within $30 \mathrm{~min}$ after administration, and his maximum blood L-DOPA concentration was $9.99 \mathrm{nmol} / \mathrm{mL}$ (dashed line). 